\title{
GRUPOS FUNCIONALES DE ÁRBOLES EN BOSQUES SECUNDARIOS DE LA REGIÓN BAJO CALIMA (BUENAVENTURA, COLOMBIA)*
}

Kelly Tatiana Bocanegra-González, ${ }^{1,2}$ Fernando Fernández-Méndez ${ }^{1,3}$, Jeferson David Galvis-Jiménez ${ }^{4}$

\section{Resumen}

El bosque húmedo tropical secundario es cada vez más común en el neotrópico y aún no se conocen sus propiedades funcionales. Los objetivos de este artículo fueron caracterizar rasgos funcionales reproductivos de especies arbóreas de bosque secundario, generar grupos funcionales y comparar diferencias existentes entre dos grados de intervención del bosque en su diversidad taxonómica y riqueza de grupos funcionales. Se establecieron seis parcelas de 0.1 ha, se muestrearon árboles con $\mathrm{DAP} \geq 10 \mathrm{~cm}$ y se caracterizaron siete rasgos funcionales reproductivos. Se encontraron 93 morfoespecies que generaron seis grupos funcionales. Se destacó la baja riqueza florística del grupo 1 en contraste con la riqueza florística de los 5 grupos restantes. Al mismo tiempo, el grupo 1 evidenció el mayor potencial funcional representado en especies conservativas y fue característico del bosque menos intervenido. Por otro lado, en el sitio con intervención permanente la redundancia funcional disminuye en este mismo grupo, lo cual puede tener consecuencias a largo plazo en la extinción local. Se concluyó que estos bosques son altamente resilientes a pesar del nivel de intervención. Sin embargo, su recuperación y preservación contribuirán a mantener su biodiversidad y la continuidad de los servicios ecosistémicos que brindan a las comunidades. Finalmente, los grupos funcionales generados pueden ser usados para planificar medidas innovadoras de manejo y restauración de áreas degradadas en la región del Chocó biogeográfico.

Palabras clave: Palabras clave: diversidad funcional, dispersión, sucesión, ensamblaje, redundancia funcional.

\section{FUNTIONAL GROUPS OF TREES IN SECONDARY FORESTS OF THE BAJO CALIMA REGION (BUENAVENTURA, COLOMBIA)}

\author{
Abstract \\ The secondary rainforest is increasingly common in the Neotropics and its \\ functional properties are unknown. The objectives of this paper were to characterize \\ reproductive functional traits of tree species of the secondary forest, generate

\footnotetext{
*FR: 2-III-2014. FA: 15-II-2015

${ }^{1}$ Ingeniera Forestal, Grupo de Investigación en Biodiversidad y Dinámica de Ecosistemas Tropicales. Universidad del Tolima. Ibagué, Colombia. E-mail: ktbocanegrag@gmail.com

${ }^{2}$ Laboratório de Conservação Genética de Espécies Florestais, Universidade Federal de Lavras -UFLA-. Lavras, Brasil.

${ }^{3}$ M.Sc., Laboratório de Ecología y Evolução de Plantas. Universidade Federal de Viçosa -UFV-. Viçosa, Brasil. E-mail: fmendez@ut.edu.co

${ }^{4}$ Ingeniero Forestal, Facultad de Ingeniería Forestal. Universidad del Tolima. Ibagué, Colombia. E-mail: jdavidgalvisj@gmail.com
} 
functional groups and compare differences between two levels of forest disturbance with its taxonomic diversity and richness in functional groups. Six plots of 0.1 ha were established, trees with $\geq 10 \mathrm{~cm}$ DAP were measured and seven functional reproductive traits were characterized. We found 93 morphospecies that generated six functional groups. Low floristic richness of group 1 in contrast to the floristic richness of the remaining 5 groups was highlighted. At the same time, group 1 showed the highest functional potential represented by conservative species and was characteristic of the least disturbed forest. On the other hand, on the site with permanent disturbance, the functional redundancy decreases in this group, which could generate to long- term local extinction. It was concluded that these forests are highly resilient despite the level of disturbance. Finally, the generated functional groups can be used to set up innovative measures in the management and restoration of degraded areas in the Chocó biogeographic region.

Key words: functional diversity, dispersion, succession, assembly, functional redundance

\section{INTRODUCCIÓN}

Los bosques tropicales están siendo sometidos a constantes intervenciones drásticas que han modificado el paisaje en muchas regiones. Los procesos de perturbación natural y antrópica afectan el funcionamiento ecosistémico de los bosques, y los impactos del cambio de uso del suelo y el cambio climático sobre la biodiversidad y productividad no están aún estimados (IPCC, 2002). Haciendo cada vez más necesario entender a diferentes niveles y escalas lo que está sucediendo con las nuevas configuraciones de vegetación que crea el hombre con su desarrollo.

Una perturbación es el cambio temporal en las condiciones ambientales medias que provoca un cambio pronunciado en un ecosistema, estas modificaciones pueden ser naturales y a menudo se producen como parte de un ciclo promoviendo la sucesión vegetal, también son causadas por los humanos y especies invasoras que no son parte del ciclo normal en determinado lugar (HUGHES, 2012). La perturbación cumple un papel fundamental en el mantenimiento de la dinámica y de la diversidad de los ecosistemas y su influencia depende de la intensidad, la localización, el tiempo y el tipo de perturbación (NEWTON, 2007).

La respuesta de los organismos o sistemas a perturbaciones según la escala en el tiempo, se manifiesta en dos procesos de respuesta del ecosistema llamados resistencia y resiliencia (FABER-LANGENDOEN, 1991; DÍAZ \& CABIDO, 2001; THOMPSON et al., 2009; DORNELAS et al., 2011; HUGHES, 2012). La pérdida de resiliencia de un ecosistema puede ser causada por la pérdida de especies o un grupo de especies con determinada función, debido a prolongadas perturbaciones que generan un cambio en la estructura física del ambiente y, por tanto, el grado de recuperación de la comunidad vegetal (CLARK \& CLARK, 1992; ASQUITH, 2002; FOLKE et al., 2004).

La forma propuesta actualmente para medir efectos y estados de diferentes sucesos sobre la biodiversidad, es estimando las funciones y la respuesta de propiedades ecosistémicas (MAGURRAN \& McGILL, 2011), lo cual se mide con la diversidad funcional (DF) definida como el valor y el rango de aquellos rasgos de especies 
y organismos que influyen en el funcionamiento de un ecosistema (TILMAN \& LEHMAN, 2002). La DF de una comunidad se puede medir de forma discontinua o continua, la primera consiste en conocer la riqueza de grupos funcionales de plantas (RGF) y la segunda es el cálculo de índices de DF (PETCHEY \& GASTON, 2006). Ambas formas se basan en caracteres ecológicamente significativos para las relaciones planta-entorno y con capacidad de reflejar la acción de diferentes presiones selectivas, estos caracteres son conocidos como rasgos funcionales (DÍAZ et al., 2002; VIOLLE et al., 2007).

La medida más común de DF es la RGF, la cual posibilita el agrupamiento de especies en una jerarquía de mayor nivel determinada por la relación con un servicio ecosistémico, función o proceso, estos agrupamientos de especies vegetales se conocen como tipos funcionales de plantas (TFP), que están constituidos por especies que desempeñan un papel semejante en el funcionamiento del ecosistema, o que representan respuestas similares a factores ambientales (DÍAZ \& CABIDO, 2001). Básicamente es agrupar las especies de acuerdo con sus respuestas o características comunes a determinados procesos para simplificar el análisis de comunidades vegetales (WESTOBY \& WRIGHT, 2006).

En el caso del proceso de resiliencia, tomado como la capacidad de un sistema para mantener su estructura y funcionamiento después de una perturbación (THOMPSON, 2011), los rasgos funcionales importantes relacionados con la capacidad de resiliencia a la perturbación son los caracteres reproductivos como la traslación de semillas por animales u otros agentes, polinización, características cualitativas como tipo, color, forma de fruto y semilla ya que brindan amplia información de esta cualidad ecosistémica, estos rasgos son importantes porque son indicativos de la recuperación y el desarrollo del bosque tras una perturbación sufrida (CASANOVES et al., 2011; THOMPSON, 2011), muestran la capacidad de reproducción y dispersión de cada especie explicando la alta biodiversidad de los bosques tropicales (CONNELL, 1978) marcando la tendencia de la sucesión vegetal a configurar ensamblajes de comunidades vegetales con características de estadios maduros con similaridad a bosques de la misma región que no han sido sometidos a perturbaciones drásticas, es decir a lo que se conoce como bosques primarios.

El Bajo Calima hace parte de la región Chocó biogeográfico en el andén Pacífico colombiano, reconocida mundialmente por su alta biodiversidad y endemismo (BERRY, 2002; RANGEL, 2004; MARTÍNEZ-HIGUERA, 2006). Cerca del 35\% de sus bosques son considerados secundarios maduros y se establecieron después del aprovechamiento a tala rasa realizado a inicios de la década de los setenta por parte de una industria papelera (MELO-CRUZ \& LOZANO-BOTACHE, 2009). Sus habitantes son grupos afrocolombianos, indígenas y colonos mestizos que derivan su sustento de la oferta ambiental de los bosques, principalmente la oferta maderera (MARTÍNEZ-HIGUERA, 2006; MELO-CRUZ \& LOZANO-BOTACHE, 2009), sin ningún tipo de regulación generando gran afectación a estas formaciones vegetales en cuanto a estructura y pérdida de la biodiversidad (RANGEL, 2004).

En Colombia existen pocas publicaciones sobre diversidad funcional y, concretamente, de evaluación de grupos funcionales en bosque húmedo tropical son inexistentes. Este enfoque se ha usado para estudiar cambios en la abundancia de rasgos o grupos funcionales en gradientes ambientales, conocer efectos de diferentes factores en el ensamblaje de comunidades vegetales (LOISELLE et 
al., 1996; DÍAZ et al., 2007; LAVOREL et al., 2008) y se propone actualmente como potencial para hacer predicciones sobre el efecto del cambio climático en ecosistemas (DÍAZ \& CABIDO, 2001). Recientemente CATELLANOS-CASTRO \& BONILLA (2011), en Colombia, evaluaron los grupos funcionales de bosque alto andino con fines de caracterización de estados sucesionales y generar estrategias de restauración ecológica.

Este artículo pretende inferir la capacidad de resiliencia de los bosques secundarios del Bajo Calima considerando la presión causada por la extracción de madera, a través de la selección y evaluación de rasgos funcionales para hacer un análisis de TFP, aportando a la discusión de diversidad y redundancia funcional. Los objetivos son: 1) Caracterizar los rasgos funcionales reproductivos de las especies de bosque húmedo tropical secundario del Bajo Calima. 2) Medir la diversidad funcional de dos bosques secundarios con dos niveles de intervención a través de la metodología de grupos funcionales, y 3) Comparar las diferencias existentes entre dos áreas de bosque secundario en cuanto a diversidad taxonómica y riqueza de grupos funcionales.

\section{MATERIALES Y MÉTODOS}

Área de estudio. El Bajo Calima tiene 65.000 ha, está localizado en la vertiente izquierda de la Cordillera Occidental y sobre la parte baja de la cuenca del río Calima a $40 \mathrm{~km}$ del municipio de Buenaventura (Valle del Cauca), con altitud de 76 msnm, temperatura promedio anual de $26,5^{\circ} \mathrm{C}$, precipitación promedio anual de $7500 \mathrm{~mm}$ y humedad relativa de 90\% (POT MUNICIPIO DE BUENAVENTURA, 2002).

Toma de información primaria. Se establecieron seis parcelas permanentes de 20x50 m (0,1 ha) en sentido norte-sur, tres parcelas en el sitio Don Sixto con coordenadas N 3 $58^{\prime} 19 "$ - W $77^{\circ} 0^{\prime} 15^{\prime \prime}$, que corresponde al bosque secundario poco intervenido, y tres en el sitio San Joaquín con coordenadas N $3^{\circ} 57^{\prime} 32.4^{\prime \prime}$ - W $77^{\circ} 0$ '4.4", que corresponde al bosque secundario muy intervenido. Para un total de 0,3 ha de muestreo en cada sitio, equivalente a 3 RAB según GENTRY (1982) que pueden describir un sitio de muestreo y acordes con las recomendaciones para muestreos locales de NEWTON (2007). El estado de intervención dado a cada bosque se hizo según el testimonio de la comunidad y datos de monitoreo forestal previos de MARTÍNEZ-HIGUERA (2006).

Todos los árboles con DAP $\geq 10 \mathrm{~cm}$ se marcaron y midieron, se hicieron colectas botánicas y de frutos, se visitaron las parcelas en total 10 épocas durante los años 2011 y 2012, las muestras fueron determinadas en la sección de árboles del Herbario TOLI en el Laboratorio de Dendrología de la Universidad del Tolima. Los frutos hallados in situ se clasificaron según el tipo, se pesaron, se midieron sus dimensiones (diámetro y longitud) y posteriormente se extrajeron las semillas, se pesaron y se midieron sus dimensiones (diámetro y longitud) siguiendo los protocolos de CORNELISSEN et al. (2003). En el caso de las especies cuyos frutos no se hallaron en campo se recurrió a información secundaria de la zona, herbarios y bases de datos, no siempre encontrando las descripciones de estas variables a nivel de especie, en esos casos se recurrió a las descripciones de estas características (tipo de fruto, peso, dimensión de fruto, peso y dimensión de semilla) a nivel de género. Procesamiento de datos. Se utilizaron los rasgos tipo de fruto, dimensión del fruto, 
peso de semilla, dimensión de semilla y síndrome de dispersión (CASANOVES et al., 2011; THOMPSON, 2011); además, se proponen para este estudio la unidad de dispersión y el peso del fruto como rasgos adicionales. Para unidad y síndrome de dispersión se consideró: la morfología de las diásporas (semillas y frutos) y la naturaleza del agente dispersor. Para unidad de dispersión se tuvo en cuenta si la dispersión se realizaba bajo la forma de unidades aisladas o acompañadas de otras estructuras como fruto (DE NOIR et al., 2002). La determinación del síndrome se hizo con el apoyo de información secundaria por especie y género, se tuvieron en cuenta únicamente mecanismos de dispersión primaria descritos por ROOSMALEN (1985), SALGADO (2007) y ARANGO et al. (2011): anemocoría (viento), autocoría (explosión y gravedad), y zoocoría en dos categorías: mamalocoría (mamíferos) y ornitocoría (aves). En el caso donde las especies tuvieran más de un mecanismo de dispersión se tomaron los dos principales.

Para las especies que no presentaron información secundaria sobre este rasgo, se utilizaron los síndromes de dispersión descritos por ROOSMALEN (1985), JUDD et al. (1999) y CORNELISSEN et al. (2003). Anemocoría: frutos y semilla con estructuras aladas y/o plumosas; mamalocoría: (fauna no voladora) frutos con arilo comestibles, aromáticos, de gran tamaño y colores amarillo, verde y café; ornitocoría: (fauna voladora) frutos con arilo comestibles, de colores brillantes naranja, rojo, blanco, negro y por lo general drupas y bayas carnosas; autocoría: frutos con pocas o ninguna adaptación evidente para la dispersión.

Análisis de datos. Se construyó una matriz especie-rasgo y se analizaron los datos mediante el software estadístico Infostat Profesional (DI RIENZO et al., 2008). Se calculó la estadística descriptiva de los rasgos y correlaciones de Pearson. Posteriormente se hizo un análisis de conglomerados jerárquicos con el método de Ward, que identifica las dos especies con menor distancia para unificarlas en primera instancia; el promedio de los RF de estas especies será quienes representen la posición del grupo, luego calcula nuevamente esta misma distancia entre dos especies para formar otro grupo o entre una especie y el grupo previamente formado, así sucesivamente hasta que todas las especies se agrupen; además, incluye información de covarianza entre RF, lo que produce grupos más diferenciados entre sí, al minimizar la varianza entre grupos y maximizar la varianza entre ellos (CASANOVES et al., 2011). Para el cálculo de la distancia se utilizó la propuesta por GOWER (1971), que utiliza la distancia euclídea para las variables continuas (peso, diámetro y longitud de semillas y frutos) y Jaccard para las variables cualitativas (unidad de dispersión, tipo de fruto y síndrome de dispersión).

Posteriormente pruebas de análisis de varianza multivariado, MANOVA (Wilks), para determinar diferencias significativas entre grupos funcionales, utilizando los rasgos continuos; además, se realizó la prueba múltiple de diferencias de medidas entre grupos de Hotelling que permitieron diferenciar los TFP. Posteriormente se usaron variables dicotómicas para identificar las categorías existentes en las variables: unidad de dispersión, tipo de fruto y tipo de dispersión, a partir de estos RF se hizo un análisis de tablas de contingencia utilizando el estadístico $\mathrm{Chi}^{2}$ para establecer asociaciones en cada uno de los casos, por último se detalló la composición de los TFP. Una vez detallados los TFP para los bosques secundarios húmedos tropicales, estos se usaron para clasificar los nuevos TFP de los dos sitios en dos grados de intervención para observar gráficamente la presencia o ausencia de especies en cada grupo funcional por sitio. 


\section{RESULTADOS}

Descripción taxonómica general. Se identificaron 33 familias, 61 géneros y 93 morfoespecies con 7 indeterminadas. El sitio 1 (Don Sixto) de menor intervención tuvo 54 especies, el sitio 2 (San Joaquín) de mayor intervención tuvo 70 (Apéndice 1). Las familias botánicas más abundantes fueron: Sapotaceae, Clusiaceae, Leguminosae, Chrysobalanaceae, Lecytidaceae y Melastomataceae. Las especies más abundantes fueron: Pradosia cuatrecasasii (Aubrév.) T.D. Penn., Eschweilera integrifolia (Ruiz \& Pav. ex Miers) R. Knuth., Guatteria cargadero Triana \& Planch., Helianthostylis sprucei Baill., Inga sp.1, Licania sp.1, Mabea chocoensis Croizat., Manilkara bidentata (A.DC.) A. Chev., Miconia ruficalyx Gleason., Oenocarpus bataua Mart., Pourouma bicolor subsp. Chocoana (Standl.) C.C. Berg y Heusden., Roucheria monsalveae A.H. Gentry., Sacoglottis ovicapa Cuatrec., Vochysia ferruginea Mart., Pouteria sp.4 y Osteophloeum platyspermum (Spruce ex A. DC.) Warb.

Evaluación de rasgos funcionales. El 60\% (56) de las especies de los bosques de los dos sitios tienen el fruto como unidad dispersante, dependiendo de un vector ajeno a la planta madre para lograr la diseminación de las semillas, mientras que el $40 \%$ (47) de las especies poseen aditamentos propios que les permiten diseminarse cuando el proceso de fructificación llega a la madurez.

El tipo de fruto más común es la drupa con el 32\% de las especies, seguido de la baya con $27 \%$, relacionados con dispersión animal. En los otros tipos de fruto se destaca la cápsula dehiscente con $21 \%$ y en menor proporción legumbre (7\%), pixidio y cápsula indehiscente (5\%) y folículo con 3\% (Tabla 1).

Tabla 1. Frecuencia del rasgo tipo de fruto en las 93 especies arbóreas de los bosques secundarios del Bajo Calima.

\begin{tabular}{llcc}
\hline Clase & Categorías & FA & FR \\
\hline $\mathbf{1}$ & Baya & 24 & 0,27 \\
$\mathbf{2}$ & Cápsula dehiscente & 19 & 0,21 \\
$\mathbf{3}$ & Cápsula indehiscente & 5 & 0,05 \\
$\mathbf{4}$ & Drupa & 30 & 0,32 \\
$\mathbf{5}$ & Folículo & 3 & 0,03 \\
$\mathbf{6}$ & Legumbre & 7 & 0,07 \\
$\mathbf{7}$ & Pixidio & 5 & 0,05 \\
\hline
\end{tabular}

Categoría $=$ Estados de la variable, FA = Frecuencia Absoluta, FR = Frecuencia Relativa.

El espectro de dispersión muestra predominancia de la dispersión por mamíferos, seguida con el mismo porcentaje (28\%) en dispersión por aves (ornitocoría) y autocoría el menor porcentaje (Figura 1A). En el bosque de menor intervención la diseminación por mamíferos corresponde al 40\%, el 34\% a la autocoría, el $20 \%$ a las aves y el 6\% por viento. Muestra marcada diferencia con el bosque de mayor intervención, aunque conserva la predominancia de mamíferos como 
vector principal, el porcentaje de especies con este síndrome asociado es menor, las especies asociadas a la autocoría también muestran gran disminución, la diseminación por aves aumenta en un 13\%. Los dos bosques son recíprocos, donde la minoría de especies en ellos cumple el traslado a través del mecanismo anemocoría (Figura 1B).

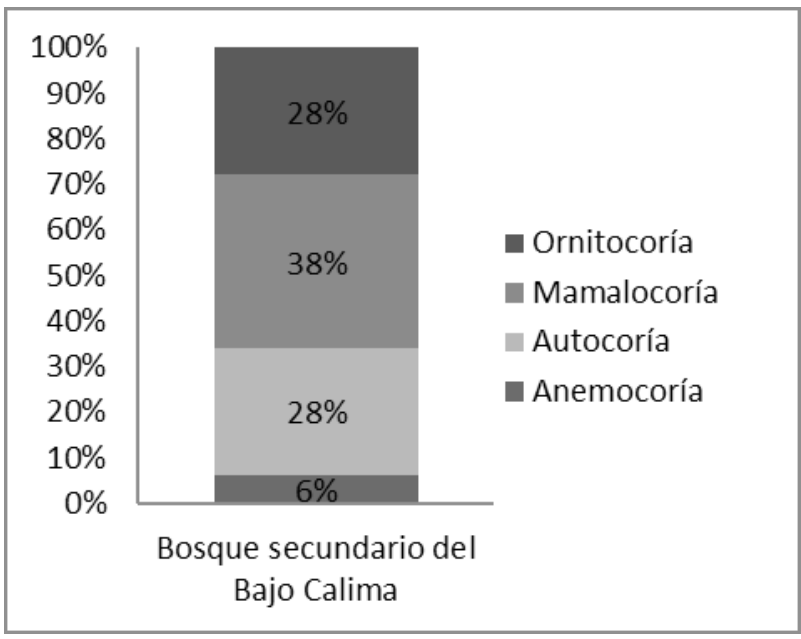

A

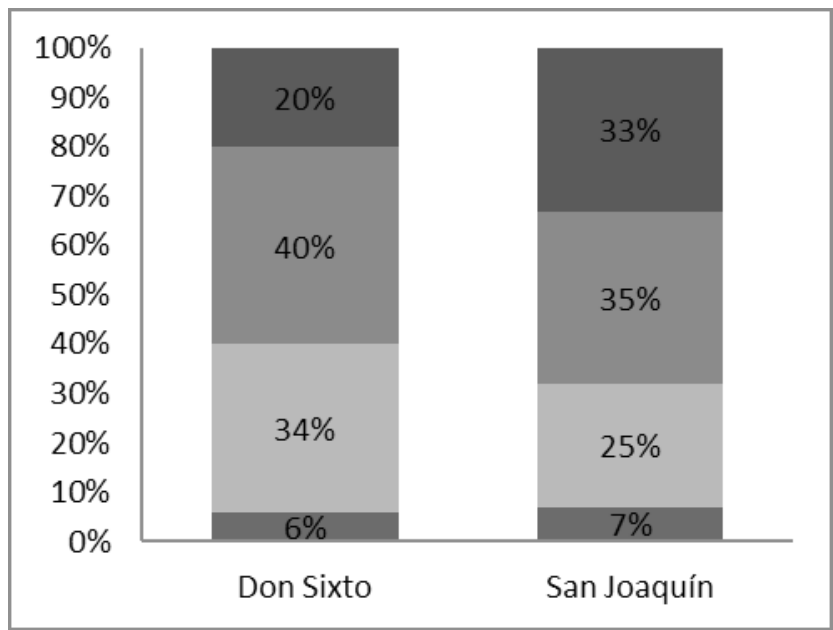

B

Figura 1. A: Espectro de dispersión para los bosques del Bajo Calima. B: Espectro de dispersión para los dos grados de intervención del bosque Don Sixto (menor grado de intervención) y bosque San Joaquín (mayor grado de intervención). 
El 79\% de los pesos de frutos que predominan son frutos livianos, entre los cuales se encuentran especies como Vismia baccifera subsp. Ferruginea (Kunth) Ewan., Guatteria calimensis R.E. Fr. y Guatteria cargadero Triana \& Planch.; y el resto (21\%) en otras corresponden a los frutos más pesados destacando especies como Theobroma bicolor Humb. y Bonpl., Carapa guianensis Aubl., Lecythis minor Jacq. El diámetro de fruto acumula el $73 \%$ con frutos menores a 3,08 cm, 10\% menores a 4,5 $\mathrm{cm}$ y el otro $17 \%$ son frutos con diámetros superiores a 5 . Debido a la correlación que existe entre peso y dimensión de fruto, las especies más representativas con valores mayores y menores fueron las mismas que se mencionan para el caso del peso. La longitud de fruto inferior a $7,8 \mathrm{~cm}$ predomino con el $89 \%$ de los frutos. Este rasgo se relaciona más con el tipo de fruto, por ejemplo las leguminosas pertenecientes a las especies Inga acreana Harms., Pentaclethra macroloba (Willd.) Kuntze. e Inga ingoides (Rich.) Willd., presentando las mayores longitudes, y las de menor longitud relacionadas con las bayas están Goupia glabra Aubl., Miconia ruficalyx Gleason. y Zanthoxylum brisasanum (Cuatrec.) P.G. Waterman. Para más detalles de la distribución de frecuencias de los rasgos de fruto se puede ver BOCANEGRA \& GALVIS (2013).

El 85 \% de las semillas que produce el bosque secundario húmedo tropical del Bajo Calima no pesan más de 2,17 g, las cuales utilizan el viento y las aves para dispersarse; y el 4\% llegan a pesar más de 8,66 g, utilizando los mamíferos y la gravedad como dispersor. El diámetro de semilla se encuentra ampliamente distribuido en cuatro clases diamétricas representadas con un 85\% que no sobrepasan un diámetro de $2,07 \mathrm{~cm}$. La longitud de semilla se encuentra distribuida en un $87 \%$ en las primeras cuatro clases que no sobrepasan $3,26 \mathrm{~cm}$ de longitud. Los diámetros y longitudes de semillas muestran una clara tendencia del bosque a dispersarse por ornitocoría y anemocoría cuando obtienen bajos valores en estas variables $y$, por el contrario, a dispersarse por mamalocoría y autocoría cuando tienen valores altos en su dimensión y peso. El 96\% de las semillas no superan una dimensión mayor de 4,78 cm. La dimensión como rasgo fue el resultado de promediar el ancho y el largo de cada semilla (Figura 2)

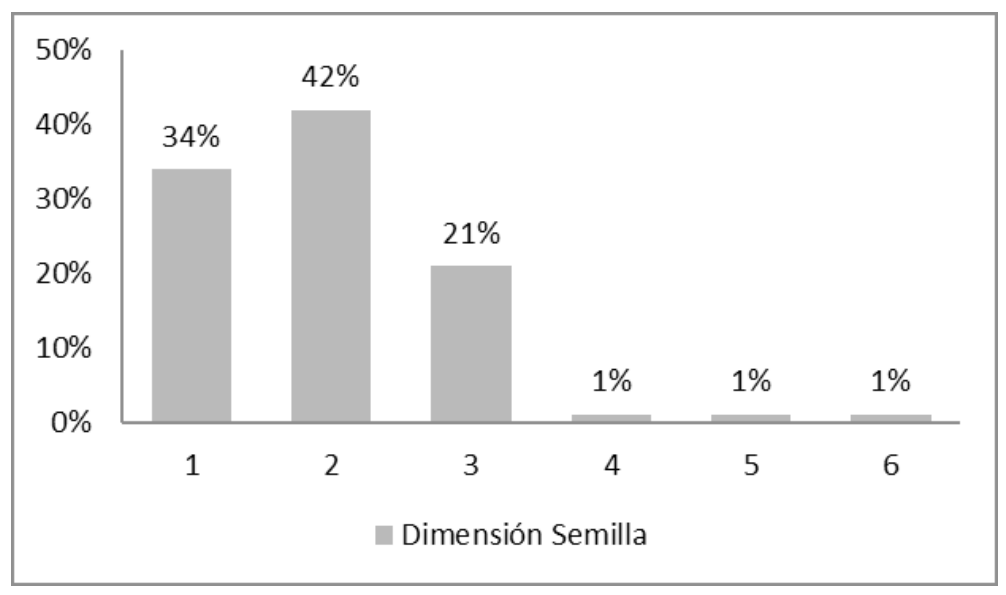

Figura 2. Distribución del rasgo dimensión de semilla en los bosques secundarios del Bajo Calima. 
El 42\% de las especies de los bosques húmedos tropicales del Bajo Calima poseen semillas con dimensiones entre $1,6 \mathrm{~cm}$ y $3,19 \mathrm{~cm}$, el $34 \%$ son especies con semillas de dimensiones mucho menores entre $0,02 \mathrm{~cm}$ y $1,6 \mathrm{~cm}$; el $21 \%$ corresponden a semillas con dimensiones mayores a las clases anteriores entre $3,19 \mathrm{~cm}$ y 4,78 $\mathrm{cm}$, y el 3\% restante corresponden a especies con semillas muy grandes mayores a $7,96 \mathrm{~cm}$ (Figura 2).

La mayor proporción de semillas en ambos sitios se encuentra en las dos primeras clases, pero Don Sixto a diferencia de San Joaquín contiene una mayor proporción y distribución de semillas en las clases de dimensión grandes. El bosque con menor intervención concentra el mayor número de especies en las dos principales que corresponden a semilla con dimensión entre $0,02 \mathrm{~cm}$ a 3,83 cm, el 81\%, el 13\% se ubican en la clase diamétrica 3 que corresponden a dimensiones entre $3,83 \mathrm{~cm}$ y $5,74 \mathrm{~cm}$; y el $6 \%$ restante en las dos clases mayores; el bosque considerado el de mayor intervención (San Joaquín) concentra el 88\% de las especies con semillas menores a 3,83 cm en las primeras clases diamétricas, tiene una representación notoria en la clase diamétrica 3 con un 10\% y casi nula en las mayores con $2 \%$ (BOCANEGRA \& GALVIS, 2013).

Según DALLING (2002) y MELO-CRUZ \& VARGAS-RÍOS (2003) una forma de caracterizar grupos ecológicos es según el tamaño de su semillas, las especies pioneras poseen semillas pequeñas al igual que las secundarias tempranas, las secundarias tardías y la especies de sucesiones maduras poseen semillas medianas y grandes, respectivamente.

Considerando lo anterior, el bosque sometido a mayor intervención (San Joaquín) posee gran ausencia de especies con tamaños de semillas correspondientes a grupos ecológicos como secundarias tardías o de bosque maduro, pero posee alta concentración de semillas pequeñas relacionadas con especies pioneras el $48 \%$. El bosque con menor intervención (Don Sixto) muestra que la mayoría de sus especies poseen semillas entre $1,92 \mathrm{~cm}$ y $3,83 \mathrm{~cm}$, lo que refiere a que no son las más diminutas pero tampoco pertenecientes a categorías mayores, se puede decir que pertenecen al grupo de secundarías tempranas y probablemente algunas de estas sean tardías (BOCANEGRA \& GALVIS, 2013).

Se observa un coeficiente de variación (CV) de 444,5\% para el peso de semillas y de $355,51 \%$ para el peso de frutos, puesto que la mayor variabilidad la aportan las especies con tipos de frutos grandes como algunos pixidios, una cápsula dehiscente y una drupa (Eschweilera reversa Pittier, Eschweilera coriácea (DC.) S.A. Mori, Lecythis minor Jacq, Licania micrantha Miq. y Carapa guianensis Aubl.), y también por tipos de frutos pequeños como es el caso de la mayoría de bayas y drupas, entre estas están todas las especies de Melastomataceae, Sapotaceae y Clusiaceae. Y para la longitud de los frutos el CV fue de 151,45\% debido principalmente a todas las especies de las familias de Caesalpiniaceae, Mimosaceae, Malvaceae, que tienen tipo de fruto en legumbre y folículo. El resto de rasgos funcionales obtuvo un CV menor del 87\% lo que indica una menor dispersión de sus valores (Tabla 2).

Se encontraron correlaciones de Pearson significativas directas entre DS y la LS ( $\mathrm{p} \leq$ 0 ), DS y DF ( $p \leq 2,3 \mathrm{E}-12$ ) y entre LS y DF ( $p \leq 2,6 \mathrm{E}-10)$, ya que las semillas guardan en diferentes proporciones las relaciones alométricas que han desarrollado buscando la mejor estrategia de protección y dispersión posible. Las relaciones entre PF y 
DF ( $p \leq 0)$, PF y LF ( $p \leq 5,0$ E-04), PF y DS ( $p \leq 1,0$ E-05), PS y DS $(p \leq 0)$, PS y LS $(p \leq$ $4,1 \mathrm{E}-08)$, fueron significativas puesto que el peso del fruto y la semilla depende de las dimensiones de las mismas, además el fruto por contener la(s) semilla(s) depende de la dimensión de esta última.

Tabla 2. Estadística descriptiva para los rasgos funcionales continuos medidos en los bosques secundarios húmedos tropicales del Bajo Calima.

\begin{tabular}{lccccc}
\hline Variable & Media & D.E. & CV & Mín & Máx \\
\hline PF $(\mathbf{g r})$ & 30,88 & 109,78 & 355,51 & 0,3 & 700 \\
DF $(\mathbf{c m})$ & 2,84 & 2,48 & 87,1 & 0,4 & 16,5 \\
LF $(\mathbf{c m})$ & 5,19 & 7,86 & 151,45 & 0,45 & 60 \\
PS (gr) & 4,38 & 19,48 & 444,5 & 0,01 & 183,23 \\
DS (cm) & 1,35 & 0,98 & 72,6 & 0,01 & 6,2 \\
LS (cm) & 1,88 & 1,21 & 64,51 & 0,01 & 6,7 \\
\hline
\end{tabular}

$\mathrm{n}$ = Número de individuos, D.E. = Desviación Estándar, CV = Covarianza, Mín = Mínimo, Máx = Máximo, $\mathrm{P}(25)$ = Percentil (25), P(75) = Percentil (75). PF: Peso de Fruto, DF: Diámetro de Fruto, LF: Largo de Fruto, PS: Peso de Semilla, DS: Diámetro de Semilla, LS: Largo de Fruto.

Tipos funcionales de plantas (TFP). Entre las especies arbóreas del bosque secundario húmedo tropical del Bajo Calima existe una clara asociación funcional según los rasgos reproductivos representada en seis grupos funcionales (Figura 3). El MANOVA (análisis de la varianza multivariado) construido a partir de los rasgos funcionales peso, longitud y diámetro de los frutos y semillas mostró diferencias significativas $(\mathrm{p}<0,0001)$ entre grupos, y la prueba múltiple de diferencias de medidas entre grupos de Hotelling encontró que el TFP1 se diferencia significativamente $(\mathrm{P}<0,05)$ del resto de TFP, mientras que el TFP3 y el TFP5 no se diferenciaron entre sí; además, los TFP 2, 4, 5 y 6 presentaron perfiles de medias que no lograron diferenciarse entre ellos. El resto de separación de los grupos fue atribuido a las variables categóricas, unidad de dispersión, tipo de fruto y tipo de dispersión, resultaron muy útiles en la separación de los grupos funcionales, puesto que presenta asociaciones significativas $(p<0,0001)$ en cada uno de los casos, en el análisis de tablas de contingencia (BOCANEGRA \& GALVIS, 2013).

El TFP1 estuvo compuesto por 22 especies, de las cuales 17 tienen como unidad dispersante la semilla y 5 el fruto, caracterizadas por tener los mayores valores en los rasgos de peso, diámetro y longitud de frutos y semillas; el tipo de fruto más común son las cápsulas dehiscentes e indehiscentes con presencia significativa de algunos pixidios y legumbres. El síndrome de dispersión de este grupo es la autocoría con algunas especies diseminadas por mamalocoría.

El TFP2 se compone de 12 especies, las cuales tienen dispersión por mamalocoría y ornitocoría, para dicho proceso usan el fruto que son bayas moderadamente grandes y pesadas como unidad de dispersión. El TFP3 dispone de 12 especies y está caracterizado por tener bayas generalmente livianas y pequeñas al igual que sus semillas, estas utilizan el fruto como unidad de dispersión a través de la ornitocoría. 


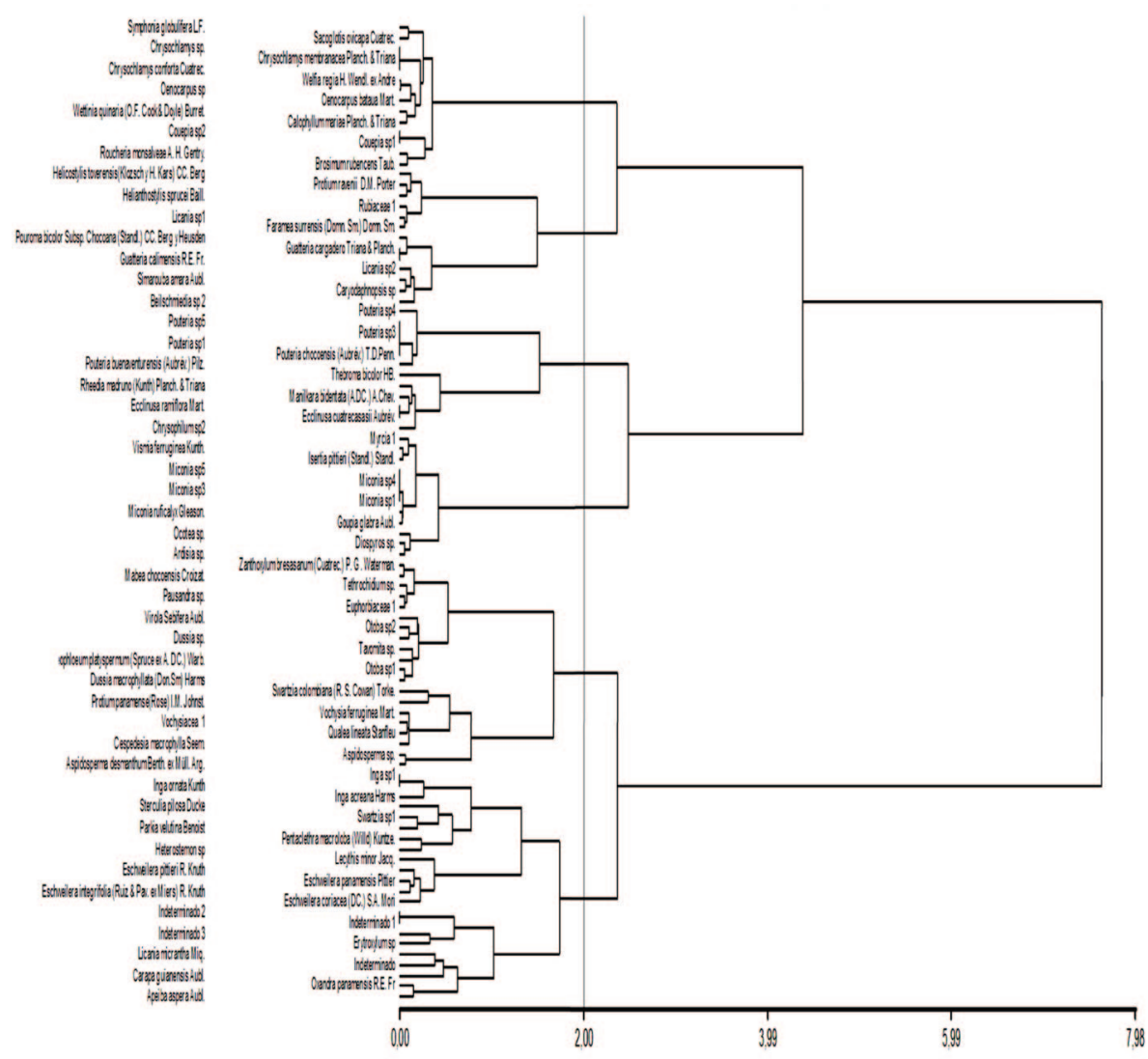

Figura 3. Tipos funcionales de árboles para las 93 especies encontradas en los bosques secundarios de la región del Bajo Calima.

El TFP4 constó de 20 especies, donde 12 presentan autocoría, 6 anemócora, una mamalocoría y una ornitocoría-mamalocoría, todas utilizando como unidad de dispersión la semilla; el tipo de fruto que domina este grupo son las cápsulas dehiscentes con 18 especies y 2 son folículos. El TFP5 está constituido por 13 especies, de las cuales 7 tienen dispersión por ornitocoría-mamalocoría y 5 mamalocoría, utilizando el fruto que son drupas de tamaño mediano como unidad de dispersión.

El TFP6 compuesto por 14 especies, de las cuales 4 son palmas todas pertenecientes a la familia Arecaceae y se caracteriza por utilizar el fruto como unidad de dispersión, estas drupas se dispersan solo por mamalocoría.

Comparación de los TFP en dos grados de intervención. Para toda la zona de estudio las especies se dividieron en 6 grupos funcionales de plantas, lo cual representa que cada grupo tiende a tener las mismas estrategias de dispersión (Figura 3) y posteriormente contrastando la composición funcional tenemos que en el sitio más conservado hay 54 especies distribuidas así: el TFP1 se compone de 16 especies en su mayoría pertenecientes a las familias Mimosaceae y Lecythidaceae; 
el TFP2 consta de 6 especies todas de la familia Sapotaceae; el TFP3 tiene 5 especies de las cuales 3 son Melastomataceae, una Lauraceae y una Myrtaceae; el TFP4 cuenta con 12 especies, que en su mayoría son Myristicaceae y Vochysiaceae; el TFP5 está compuesto de 6 especies con 2 Chysobalanaceae y una respectivamente de las familias Annonaceae, Burseraceae, Moraceae y Urticaceae; finalmente el TFP6 está compuesto por 9 especies, 2 Arecaceae, 2 Chysobalanacae, 2 Clusiaceae y una especie respectivamente de las familias Humiriaceae, Linaceae y Moraceae (Tabla 3 y Figura 4).

Tabla 3. Composición florística de cada TFP en diferentes tipos de bosque de la región del Bajo Calima.

\begin{tabular}{lcccccc}
\hline Tipos de bosques & TFP1 & TFP2 & TFP3 & TFP4 & TFP5 & TFP6 \\
\hline Bosque Secundario & 22 & 12 & 12 & 20 & 13 & 14 \\
Bosque Secundario & 16 & $6(50 \%)$ & 5 & 12 & 6 & $9(64,2 \%)$ \\
Conservado & $(72,7 \%)$ & & $(41,6 \%)$ & $(60 \%)$ & $(46,15 \%)$ & 10 \\
Bosque Secundario & 10 & 11 & 11 & 16 & 12 & 10 \\
Intervenido & $(45,4 \%)$ & $(91,6 \%)$ & $(91,6 \%)$ & $(80 \%)$ & $(92,3 \%)$ & $(71,42 \%)$ \\
\hline
\end{tabular}

Grupos Funcionales = TFP1, TFP2, TFP3, TFP4, TFP5, TFP6.

Para el caso del bosque más intervenido, San Joaquín, el dendrograma se compone de 70 especies. Encontrando mayor riqueza en los TFP a excepción del grupo de especies con frutos grandes. El TFP1 se compuso de 10 especies, donde dos fueron Lecythidaceae y Malvaceae, el resto con familias diferentes, Annonaceae, Chrysobalanaceae, Lauraceae, Meliaceae, Mimosaceae, Papilionaceae; el TFP2 consta de 11 especies, con 9 Sapotaceae, una Clusiaceae y una Malvaceae; el TFP3 presentó 11 especies, 5 Melastomataceae, una Ebenaceae, una Goupiaceae, una Hypericaceae, una Primulaceae, una Myrtaceae y una Rubiaceae; el TFP4 con 16 especies, es el que más riqueza tiene, distribuidas en 8 familias, Myristicaceae, Euphorbiaceae, Apocynaceae, Papilonaceae, Vochysiaceae, Burceraceae, Ochnaceae y Rutaceae; El TFP5 cuenta con 12 especies, repartidas en diferentes familias, 2 Annonaceae, 2 Lauraceae, 2 Moraceae, 2 Rubiaceae, una Burseraceae, una Chrysobalanaceae, una Simaroubaceae y una Urticaceae; y el TFP6 con 10 especies, 4 Clusiaceae, 3 Aracaceae, una Humiriaceae, una Linaceae y una Moraceae (Tabla 3 y Figura 5).

Se observa que el bosque conservado presenta un $72 \%$ de las especies del TFP1, mucho mayor que en el bosque San Joaquín, caracterizado por especies de boque maduro. Además, en cuanto al TFP2 y al TFP3, se observa una fuerte disminución en su riqueza, contrario a lo que ocurre en el bosque intervenido puesto que estos grupos se caracterizan por ser muy eficientes en su dispersión para así poder colonizar los claros que se generan en el bosque por la intervención ya mencionada; simultáneamente los TFP 4, 5 y 6, poseen características de grupos ecológicos primarios y secundarios, ya que tienen pesos y tamaños de frutos y semillas variados al igual que sus tipos de frutos, aparte de dispersarse generalmente por animales o por gravedad. Para el caso del bosque más intervenido, San Joaquín, el dendrograma se compone de 70 especies; se encontró que la riqueza funcional en cada TFP es mayor en comparación del bosque conservado, lo cual presenta una alta redundancia funcional en los grupos exceptuando el TFP1. 

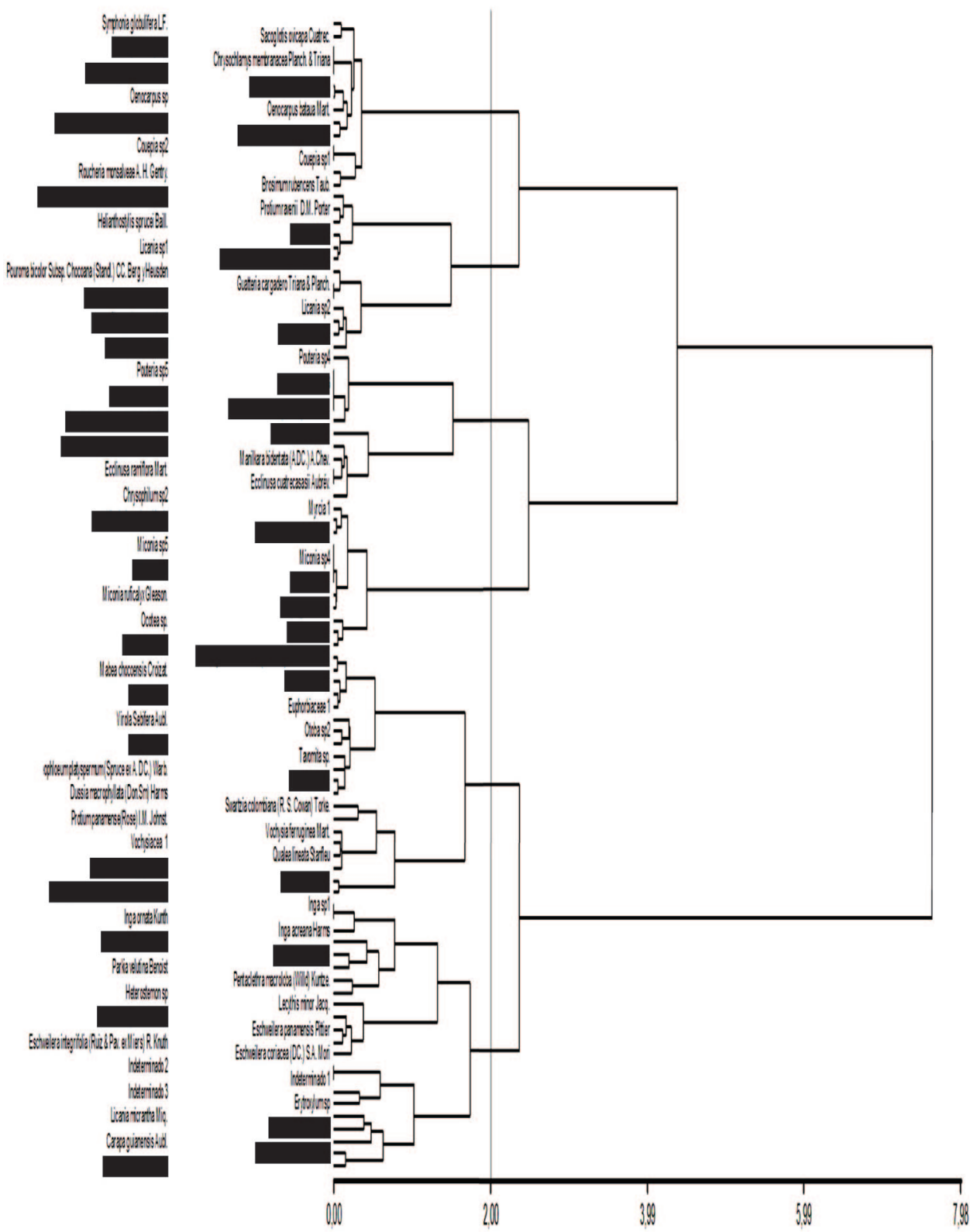

Figura 4. Tipos funcionales de plantas para el bosque secundario conservado en la región del Bajo Calima. 


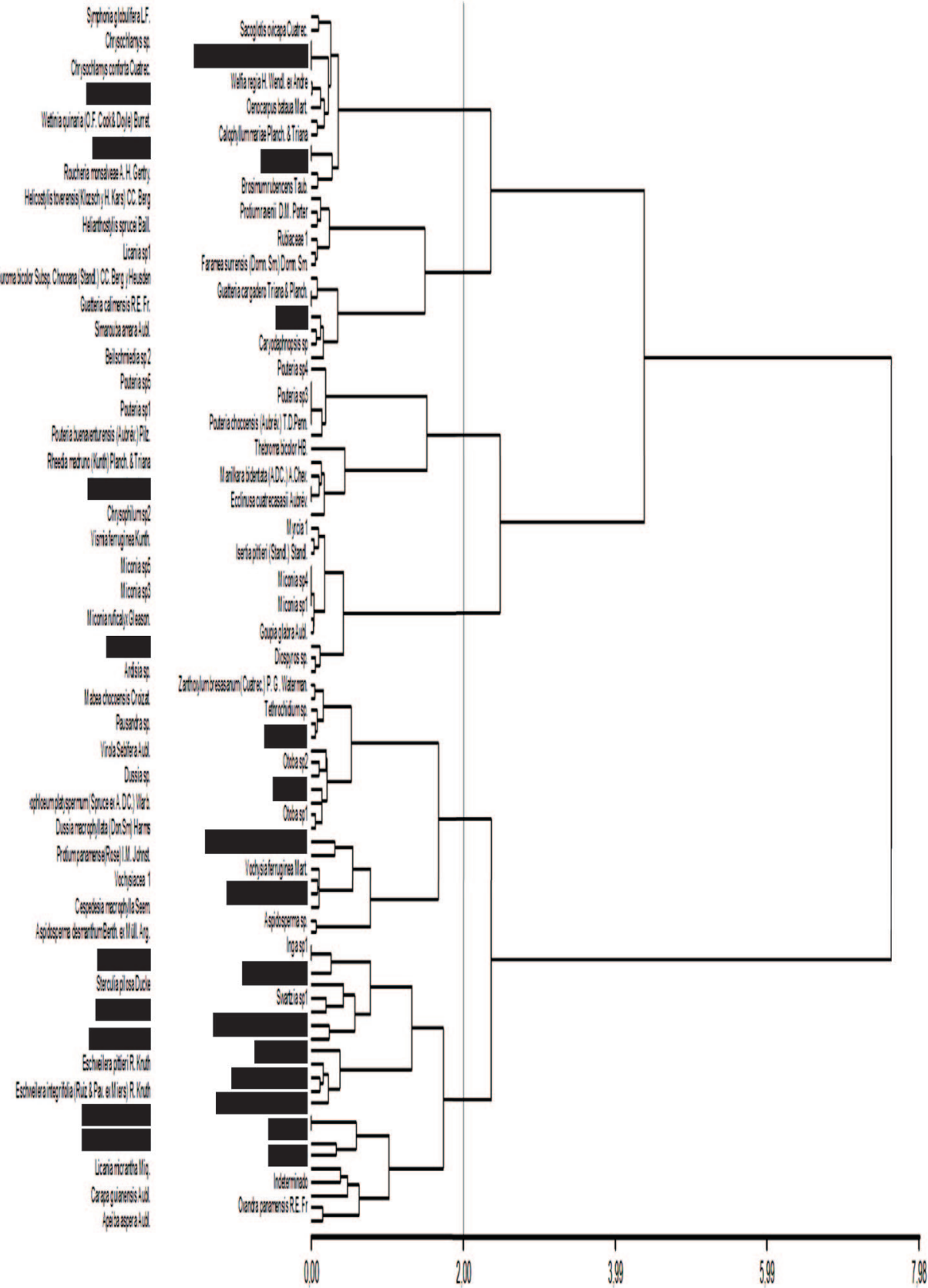

Figura 5. Tipos funcionales de plantas para el bosque secundario intervenido en la región del Bajo Calima. 
El TFP1 se compuso de 10 especies, 2 pertenecientes a la familia Lecythidaceae y Malvaceae, el resto de especies pertenecientes a las familias Annonaceae, Chrysobalanaceae, Lauraceae, Meliaceae, Mimosaceae, Papilionaceae; el TFP2 consta de 11 especies, 9 Sapotaceae, una Clusiaceae y una Malvaceae; el TFP3 presentó 11 especies, 5 Melastomataceae, y una especie respectivamente de las familias Ebenaceae, Goupiaceae, Hypericaceae, Primulaceae, Myrtaceae y Rubiaceae; el TFP4 con 16 especies, es el que más riqueza tiene, distribuidas en 8 familias, Myristicaceae, Euphorbiaceae, Apocynaceae, Papilionaceae, Vochysiaceae, Burseraceae, Ochnaceae y Rutaceae; el TFP5 cuenta con 12 especies, 2 especies en cada una de las familias Annonaceae, Lauraceae, Moraceae, Rubiaceae y una especie respectivamente en las familias Burseraceae, Chrysobalanaceae, Simaroubaceae y Urticaceae. Por último, el TFP6 con 10 especies, 4 Clusiaceae, 3 Arecaceae, y una especie por cada una de las familias Humiriaceae, Linaceae y Moraceae.

Además, en el TFP2 y el TFP3 se observa una fuerte disminución en su diversidad, contrario a lo que ocurre en el bosque San Joaquín puesto que estos grupos se caracterizaron por tener mayor número de especies y poseer características que las hacen más eficientes en su dispersión, esta cualidad les permite colonizar los claros que se generan en el bosque por la intervención producida por la extracción de madera tradicional; simultáneamente, los TFP4, 5 y 6 poseen características de grupos ecológicos primarios y secundarios, ya que sus rasgos de peso y tamaño de frutos y semillas, tipo de fruto y tipo de dispersión son variados.

\section{DISCUSIÓN}

Es importante destacar una fuerte dependencia de vectores animales para promover el proceso de diseminación y regeneración de las especies vegetales del bosque estudiado. Se analizaron dos bosques secundarios con diferentes grados de intervención antrópica, referida únicamente a la extracción de maderera. En el caso del bosque con mayor intervención (San Joaquín) el número de especies fue mayor que el bosque tratado como más conservado o con menor grado de intervención (Don Sixto). CONNELL (1978) a través de la teoría de la perturbación intermedia sostiene que en ecosistemas maduros la presencia de perturbaciones intermedias permite mantener niveles de riqueza de especies y, por tanto, de biodiversidad mayor de los que habría en ausencia de estas perturbaciones. ROXBURGH et al. (2004) demuestran que estas alteraciones articulan diferentes mecanismos como los fenómenos de dispersión, de recolonización, de competencia, que generan el aumento de la diversidad partiendo de un proceso de perturbación. Desde el punto de vista taxonómico es común este comportamiento en áreas recién colonizadas con buenas fuentes de semillas como la región de estudio.

No existe información sobre los patrones de sistemas de dispersión de plantas en las distintas regiones geográficas del país (CORREA-GÓMEZ et al., 2013). A pesar de que se ha avanzado en trabajos de caracterización importantes de diferentes ecosistemas colombianos (RANGEL, 2004), se requiere más conocimiento del comportamiento funcional.

En regiones tropicales se ha reportado una alta frecuencia de especies dispersadas por animales, mayor a la encontrada en regiones templadas (GENTRY, 1982) y el área de estudio no es la excepción, donde predominan en el bosque conservado 
las especies dispersadas por mamíferos y aves. El ensamblaje funcional de los distintos sistemas de dispersión cambian cuando las comunidades y las poblaciones de frugívoros son afectadas por la cacería o por la fragmentación de los bosques (WRIGHT, 2003). Tal como se muestra en el presente estudio, donde la dominancia de especies dispersadas por animales es mayor en el bosque secundario conservado.

Los TFP de plantas son el resultado de respuestas similares al ambiente o efectos similares en los procesos ecosistémicos donde se resume la enorme diversidad de especies en grupos más o menos predecibles (BLONDEL, 2003; DÍAZ et al., 2002). Considerando esto, la respuesta del bosque secundario húmedo tropical del Bajo Calima al proceso ecosistémico resiliencia se puede reducir en 6 TFP, donde se encontraron especies generalmente con valores mayores en tamaño y peso de frutos y semillas, por lo que un 24,73\% de las especies del bosque se agruparon en el TFP1, el resto de TFP poseen menores pesos y tamaños. El tamaño y peso de fruto y semilla dependen de cómo es el ambiente que los rodea y de cómo la semilla se ha adaptado a esas condiciones; el tamaño probablemente representa un compromiso entre los requerimientos de dispersión (que favorecerían tamaños menores) y las necesidades de reservas que utiliza la plántula para establecerse (que favorecerían tamaños mayores); en consecuencia, el peso y tamaño de los frutos y semillas son un índice relativo de la inversión de materia y energía que hace la planta madre para desarrollarlos (DALLING \& HUBBELL, 2002), lo cual también es conocido como trade-off o estrategias de las plantas.

La producción de un fruto y semilla grande podría implicar que el número de semillas sea mínima, o que sea necesario un periodo largo entre cosechas, o que la provisión de buenas reservas aumente la probabilidad de permanecer más tiempo en el suelo antes de independizarse, puesto que la competencia en estos bosque es alta, en estas condiciones la dispersión es menos importante que contar con el tiempo suficiente para que la planta desarrolle raíces y hojas bien formadas antes de independizarse, además de favorecer la germinación cuando estas quedan enterradas, generalmente estas especies toleran la sombra (DALLING \& HUBBELL, 2002); esto favorece relativamente a la resiliencia puesto que asegura la existencia de una especie después de una perturbación fuerte. Por otro lado, los frutos y semillas pequeñas y livianas pueden producirse en mayores cantidades y tener gran capacidad de dispersión, asegurando que por lo menos algunas cuantas logren colonizar nuevos claros y asegurar su existencia (DALLING, 2002). Se observa entonces una alta redundancia en los grupos funcionales, lo que da como resultado una buena resiliencia ya que se asume que la existencia de al menos un miembro de un grupo funcional garantiza su función o su respuesta (WALKER, 1992).

El rasgo unidad de dispersión dividido en dos características estructurales principales, logró separar dos grandes grupos, uno que utiliza las semillas como unidad de dispersión y el resto de TFP que usan el fruto como medio dispersor; el TFP1 con tipos de frutos en cápsulas dehiscentes e indehiscentes, legumbres y pixidios principalmente, es dispersado por gravedad, mamíferos y aves; y el TFP4 con frutos en cápsulas dehiscentes y dos folículos, está dispersado por el viento, la gravedad y en menor proporción por mamíferos y aves.

Los mecanismos de dispersión representan un rasgo muy importante para la recolonización después de las perturbaciones y para favorecer el flujo genético dentro del paisaje (DALLING, 2002), puesto que la regeneración de un bosque 
depende de la capacidad de dispersión de las semillas y estas a las condiciones de hábitat a las que van llegar. Investigaciones han determinado que las semillas dispersadas por el viento (anemócoras) llegan en mayor número a los claros, al ser favorecidas por los movimientos del aire. Sin embargo, las semillas con otras formas de dispersión, como las autócoras y zoócoras, parecen ser poco influenciadas por los claros (LOISELLE et al., 1996).

Para clasificar las especies de cada sitio en TFP se utilizó el modelo de grupos funcionales general del bosque secundario muy húmedo tropical del Bajo Calima (BOCANEGRA \& GALVIS, 2013), puesto que este contiene implícito en cada TFP las especies con características iguales en toda la región de estudio. En donde una mayor diferencia en la composición florística entre cada TFP por sitio y los TFP del bosque en general, exhiben una ausencia de parte de su riqueza funcional, y la pérdida de un TFP completo sugiere una pérdida en su capacidad de resiliencia, y a la vez estados sucesionales diferentes. Asumiendo que a mayor riqueza en cada uno de los TFP, mayor capacidad tendrá de absorber disturbios el bosque secundario, y así poder retener sus funciones y estructura básica (WALKER, 1995). Conservando en el mosaico de la región una alta diversidad de especies, funcional y eventualmente genética que debe ser objeto de estudio.

En cuanto al TFP1, la mayor diversidad florística la presenta el bosque Don Sixto que es el menos intervenido, esto respalda la condición de este bosque como el más conservado, puesto que las especies que alberga tienen características como dimensión de la semilla y tipo de dispersión típicas del grupo ecológico de las secundarias y pertenecen a las familias Chrysobalanaceae, Lecythidaceae, Meliaceae, y Mimosaceae, Caesalpiniaceae, tal como las clasifica MARTÍNEZ-HIGUERA (2006).

Las diferencias en cuanto a diversidad florística en los TFP2 y 3 son mucho menores en el bosque San Joaquín, por ser grupos con altas probabilidades de dispersar sus bayas generalmente pequeñas y livianas por medio de aves y mamíferos en el TFP2 y por aves en el TFP3, estas características inducen gran capacidad de dispersión, lo que permite recorrer relativamente largas distancias para colonizar claros. Estos grupos están compuestos por especies que en su mayoría pertenecen al grupo ecológico de las primarias o pioneras porque se establecen y crecen en claros recientes, como los que se generan después de un aprovechamiento o de la caída natural de un árbol. Estas especies pertenecen a las Melastomataceae y Sapotaceae, y solo dos familias con una especie cada una pertenecen al grupo ecológico de las secundarias, Myrtaceae y Lauraceae según MARTÍNEZ-HIGUERA (2006).

San Joaquín cuenta con una mayor riqueza funcional en los TFP4, 5 y 6 que Don Sixto (Tabla 3), aunque las diferencias entre estas dos no son tan marcadas, además por ser estos bosques tan diversos se plantea una redundancia funcional dentro de cada TFP que no permite que el proceso ecosistémico de resiliencia se afecte a grandes escalas. Estos grupos están caracterizados por contener drupas dispersadas por aves y mamíferos, y por la gravedad, el tamaño y peso de estos frutos y semillas en promedio son moderadamente grandes lo que indica que existen especies pertenecientes a los dos grupos ecológicos. El primer grupo es de especies tolerantes a la luz pertenecientes a familias como Annonaceae, Burseraceae, Clusiaceae, Melastomataceae, Meliaceae, Ochnaceae, Rubiaceae, Simaroubaceae y Vochysiaceae; y las especies tolerantes a la sombra de familias como: Caesalpiniaceae, Chrysobalanaceae, Apocynaceae, Euphorbiaceae, Humiriaceae, 
Lauraceae, Lecythidaceae, Linaceae, Mimosaceae, Moraceae, Myristicaceae, Myrsinaceae, Papilionaeae, Sapotaceae y Sterculiaceae (MARTÍNEZ-HIGUERA, 2006 El TFP más importante para la evaluación de la resiliencia fue el TFP1 por contener especies del grupo ecológico de las esciófitas con frutos y semillas grandes y de gran peso, dispersadas por gravedad y animales, lo que indica un alto grado de conservación; este grupo funcional es representativo en Don Sixto. Por otro lado el TFP2 y el TFP3 contienen frutos y semillas livianas de pequeños tamaños, con dispersión por aves o viento, lo que aumenta su probabilidad de dispersión, ubicado las especies de estos TFP en el grupo ecológico de las heliófilas; estos grupos funcionales son representativos en San Joaquín por la constante intervención que recibe el bosque. En cuanto a los TFP4, 5 y 6, al presentar frutos y semillas moderadamente pesadas y grandes que se relacionan con síndromes de dispersión variados, indican cierto grado de desarrollo en los dos bosques con distinto grado de intervención.

Los resultados de este estudio demuestran que tanto el bosque con menor intervención y el bosque de mayor intervención presentan resiliencia potencial a diferentes escalas. El primero tiene una resiliencia más lenta en términos de establecimiento y crecimiento de individuos, puesto que estas especies requieren de tiempo suficiente y una oportunidad en el bosque para emerger, establecerse y desarrollarse, ya que estas van a ser especies duraderas en el tiempo. Por el contrario, la segunda posee especies de crecimiento rápido lo que las ubica en el grupo ecológico de las pioneras, las cuales cuentan con estrategias efectivas para establecerse en los claros que van quedando en el bosque después de su intervención pero son poco duraderas en el tiempo, este tipo de bosque tiene una regeneración constante y rápida, lo que permite que exista una gran diversidad de especies forestales.

A escala del paisaje de la región se muestra que aún se conservan interacciones con fauna que permiten una alta diversidad tanto en los sitios conservados como en los que son utilizados para sacar madera de pequeñas dimensiones, lo cual va moldeando el ensamblaje de la comunidad arbórea y modificando la relación tamaño de semilla y establecimiento, destacada por FOSTER \& JANSON (1985) como importante para entender el funcionamiento del ecosistema. Estos cambios en la reproducción y la composición funcional de los ecosistemas muy intervenidos han sido ampliamente comprobados como en el caso del bioma de Mata Atlántica de Brasil (GIRÃO et al., 2007).

Entender esta proporción funcional de la unidad de dispersión es fundamental a la hora de entender la sucesión y plantear medidas de conservación, ya que la morfología de estos facilita o dificulta el transporte y posterior establecimiento de la semilla, configurando la composición de los TFP, de acuerdo a las interacciones con los dispersores. ARANGO et al. (2011) y MANIGUAJE et al. (2011) sugieren que los tipos de frutos que están relacionados con los mecanismos de dispersión animal tienen una mayor distancia de dispersión y menor agregación, lo cual los hace más vulnerables y por consiguiente objetos de conservación si se quiere aumentar resiliencia.

Evidentemente en el sitio con intervención permanente la redundancia funcional se pierde en un grupo, lo cual puede tener consecuencias a largo plazo en extinción local (LAWTON \& BROWN, 1993). Dicha redundancia es la que sostiene el 
funcionamiento de los ecosistemas forestales en las diferentes funciones y procesos en los que interviene cada especie (WALKER et al., 1999).

Es importante reconocer que estos bosques son altamente resilientes y que si se dejan recuperar lograrán mantener alta biodiversidad gracias a la cercanía a áreas más conservadas y la presencia de muchos dispersores como aves y mamíferos, de los cuales se han reportado recientemente 85 especies de aves y 21 de mamíferos para esta región según QUIMBAYO (2006).

MULLER-LANDAU (2010) toma las características tamaño y fertilidad como uno de los principales trade-off para separar especies en un rango de comportamientos frente a la sucesión, este comportamiento de trade-off se ve reflejado en la comparación de los dos sitios evaluados, encontrando predominancias del grupo funcional 1 en el bosque conservado donde existe menos luminosidad y la tendencia a la ausencia en el bosque intervenido. Finalmente, no se descarta posibilidades de que estos bosques sean manejados gracias a su alta resiliencia evidenciada en este estudio, lo que permite pronosticar que estos bosques secundarios tienen potencial de manejo sostenible tal como lo recomienda FINEGAN (1992), a partir de bosques secundarios en América Central, los cuales tienen estructuras y funcionamientos similares a los del Chocó biogeográfico.

GUARIGUATA \& OSTERTAG (2002) muestra cómo las sucesiones secundarias pueden alcanzar propiedades similares a los bosques primarios en cuanto a estructura y biomasa, y destaca que la recuperación funcional en cuanto a biodiversidad y relaciones con dispersores es un proceso mucho más largo en la escala temporal. Estos estudios de otras regiones muestran cómo el Bajo Calima tiene gran potencial para la recuperación de sus bosques y poder seguir prestando servicios ecosistémicos a las comunidades que allí habitan.

\section{AGRADECIMIENTOS}

Los autores desean agradecer al fondo de investigaciones de la Universidad del Tolima por financiar esta investigación, al Consejo Comunitario del Bajo Calima por permitir la realización de este trabajo en su territorio, al Centro Forestal Tropical Pedro Antonio Pineda por brindar las condiciones necesarias, a los guías de campo y al Laboratorio de Dendrología de la Universidad del Tolima.

\section{BIBLIOGRAFÍA}

ARANGO, H., DUQUE, A., CÁRDENAS, D. \& BARRETO, J., 2011.- Relación entre el mecanismo de dispersión de semillas y distribución de algunas especies arbóreas en un bosque de tierra firme de la Amazonia colombiana. Colombia amazónica, 4: 87-96.

ASQUITH, N.M., 2002.- La dinámica del bosque y la diversidad arbórea: 377-406 (en) GUARIGUATA, M.R. \& KATTAN, G.H (eds.) Ecología y conservación de bosques neotropicales. Editorial Tecnológica de Costa Rica, Turrialba, Costa Rica.

BERRY, E., 2002.- Diversidad y endemismo en los bosques neotropicales de bajura: 13-32 (en) GUARIGUATA, M.R. \& KATTAN, G.H. (eds.) Ecología y conservación de bosques neotropicales. Editorial Tecnológica de Costa Rica, Turrialba, Costa Rica.

BLONDEL, J., 2003.- Guilds or functional groups: does it matter? Oikos, 100: 223-231.

BOCANEGRA GONZÁLEZ, K.T. \& GALVIS JIMÉNEZ, J.D., 2013.- Determinación de la resiliencia en bosques secundarios húmedos tropicales a través de la diversidad funcional de árboles en la región del Bajo Calima, Buenaventura, Valle del Cauca: Tesis, Universidad del Tolima, Facultad de Ingeniería Forestal, Ibagué. 
CASANOVES, F., PLA, L. \& DI RIENZO, J.A., 2011.- FDiversity: a software package for the integrated analysis of functional diversity. Methods in Ecology \& Evolution, 2: 233-237.

CASTELLANOS-CASTRO, C. \& BONILLA, M.A., 2011.- Grupos funcionales de plantas con potencial uso para la restauración en bordes de avance de un bosque altoandino. Acta Biol. Colomb., 16 (1): 153-174.

CLARK, D.A \& CLARK, D.B., 1992.- Life history diversity of canopy and emergent trees in a neotropical rain forest. Ecological Monograph., 62 (3): 315-344.

CONNELL, J.H., 1978.- Diversity in tropical rain forests and coral reefs. Science, 199: 1302-1310.

CORNELISSEN, J.H.C., LAVOREL, S., GARNIE, E., DÍAZ, S., BUCHMANN, N., GURVICH, D.E. et al., 2003.- A handbook of protocols for standardised and easy measurement of plan functional traits worldwide. Australian Journal of Botany, 51 (4): 335-380.

CORREA-GÓMEZ, D.F., Stevenson, P., Álvarez, E., Aldana, A., Umaña, M., Cano, A. et al., 2013.- Patrones de frecuencia y abundancia de sistemas de dispersión de plantas en bosques colombianos y su relación con las regiones geográficas del país. Colombia Forestal, 16 (1): 33-51.

DALLING, J.W., (2002).- Ecología de semillas (en) GUARIGUATA, M.R. \& KATTAN, G.H. (eds.) Ecología y conservación de bosques neotropicales. Editorial Tecnológica de Costa Rica, Turrialba, Costa Rica.

DALLING, J.W. \& HUBBELL, S.P., 2002.- Seed size, growth rate and gap microsite conditions as determinants of recruitment success for pioneer species. Journal of Ecology, 90 (3): 557-568.

DE NOIR, F., BRAVO, S. \& ABDALA, R., 2002.- Mecanismos de Dispersión de Algunas Especies Leñosas Nativas del Chaco Occidental y Serrano. Quebracho, 9: 140-150.

DÍAZ, S. \& CABIDO, M., 2001.- Vive la difference: Plant functional diversity matters to ecosystem processes. Trends in Ecology and Evolution, 16 (11): 646-654.

DÍAZ, S., GURVICH, D., PÉREZ, H.N. \& CABIDO, M., 2002.- ¿Quién necesita tipos funcionales de plantas? Boletín de la Sociedad Botánica de Argentina, 37 (2): 135-140.

DÍAZ, S., LAVOREL, S., DE BELLO, F., QUÉTIER, F., GRIGULIS, K. \& ROBSON, M., 2007.- Incorporating plant functional diversity effects in ecosystem service assessments. PNAS, 104 (52): 20684-20689.

DI RIENZO, J.A., CASANOVES, F., BALZARINI, M.G., GONZÁLEZ, L., TABLADA, M. \& ROBLEDO, C.W., 2008. Software InfoStat. Grupo InfoStat, FCA, Universidad Nacional de Córdoba, Argentina.

DORNELAS, M., PHILLIP, D.A.T. \& MAGURRAN, A.E., 2011.- Abundance and dominance become less predictable as species richness decreases. Global Ecology and Biogeography, 20 (6): 832-841.

FABER-LANGENDOEN, D., 1991.- Ecological constraints on rain forest management at Bajo Calima, western Colombia. Forest Ecology and Management, 53: 213-244.

FINEGAN, B., 1992.- El potencial de manejo de los bosques húmedos secundarios neotropicales de las tierras bajas. CATIE. Serie Técnica. Informe Técnico Colección Silvicultura y Manejo de Bosques Naturales, 188 (5).

FOLKE, C., CARPENTER, S., WALKER, B., SCHEFFER, M., ELMQVIST, T., GUNDERSON, L. \& HOLLING, C.S., 2004.- Regime shifts, resilience, and biodiversity in ecosystem management. Annual Review of Ecology, Evolution and Systematics, 35: 557-581.

FOSTER, S.A. \& JANSON, C.H., 1985.- The relationship between seed size and establishment conditions in tropical woody plants. Ecology, 66: 773-780.

GENTRY, A.H., 1982.- Patterns of neotropical plant species diversity. Evolutionary Biology, 15: 1-84.

GIRÃO, L.C., LOPES, A.V., TABARELLI, M. \& BRUNA, E.M., 2007.- Changes in Tree Reproductive Traits Reduce Functional Diversity in a Fragmented Atlantic Forest landscape. PLoS ONE, 2 (9): E908.

GOWER, J.C., 1971.- A general coefficient of similarity and some of its properties. Biometrics, 27: 857-874.

GUARIGUATA, M.R. \& OSTERTAG, R., 2002.- Sucesión secundaria: 377-406 (en) GUARIGUATA, M.R. \& KATTAN, G.H. (eds.) Ecología y conservación de bosques neotropicales. Editorial Tecnológica de Costa Rica, Turrialba, Costa Rica.

HUGHES, A., 2012.- Disturbance and Diversity: An Ecological Chicken and Egg Problem. Nature Education Knowledge, 3 (10): 48.

IPCC, INTERGUBERNAMENTAL PANEL OF CLIMATE CHANGE., 2002.- Climate change and biodiversity. IPCC Technical paper $V$. IPCC-WMO-UNEP.

JUDD, W.S., CAMPBELL, C.S., KELLOGG, E.A. \& STEVENS, P.F., 1999.- Plant Systematics: A Phylogenetic Approach. Sinauer Associates, Inc., Sunderland, Massachusetts, USA.

LAVOREL, S., GRIGULIS, K., MCINTYRE, S., WILLIAMS, N.S.G., GARDEN, D., DORROUGH, J. et al., 2008. Assessing functional diversity in the field - methodology matters! Functional Ecology, 16: 134-147.

LAWTON, J.H. \& BROWN, V.K., 1993.- Redundancy in ecosystems: 255-270 (en) SCHULZE, E.D. \& MOONEY, H. (eds.) Biodiversity and ecosystem function. Springer, Berlin.

LOISELLE, B.A., RIBBENS, E. \& VARGAS, O., 1996.- Special and temporal variation of seed rain in a tropical lowland wet forest. Biotropica, 28: 82-95.

MAGURRAN, A. \& McGILL, B.J., 2011.- Challenges and opportunities in the measurement and assessment of biological diversity (en) MAGURRAN, A. \& McGILL, B.J. (eds.) Biological diversity: frontiers in measurement and assessment.

MANIGUAJE, L., DUQUE, A., CÁRDENAS, D. \& MORENO, F., 2011.- Changes in seed dispersal spectrum along the altitudinal gradient between wet amazonian and andean forest in Colombia. Colombia Amazónica, 4: 35-42. 
MARTÍNEZ-HIGUERA, H., 2006.- Análisis ecológico silvicultural, con fines de manejo, del bosque secundario de la vereda Las Brisas, Buenaventura, Valle del Cauca, Colombia: Tesis Doctorado en Ciencias: Biología, Universidad del Valle, Facultad de Ciencias Naturales y Exactas, Cali, Colombia.

MELO-CRUZ, O.A. \& LOZANO-BOTACHE, L.A., 2009.- Los bosques secundarios del trópico húmedo colombiano: caso del Bajo Calima. Características y expectativas para el manejo sostenible y conservación de los ecosistemas boscosos del Bajo Calima, Valle del Cauca, Buenaventura, Colombia. Universidad del Tolima, Facultad de Ingeniería Forestal, Ibagué, Colombia.

MELO-CRUZ, O.A. \& VARGAS-RÍOS, R., 2003.- Evaluación ecológica y silvicultural de ecosistemas boscosos. Universidad del Tolima, CRQ, CARDER, CORTOLIMA, CORPOCALDAS. Impresiones Conde, Ibagué, Colombia.

MULLER-LANDAU, H.C., 2010.- The tolerance-fecundity trade-off and the maintenance of diversity in seed size. PNAS, 107 (9): 4242-4247.

NEWTON, A.C., 2007.- Forest Ecology and Conservation. A Handbook of Techniques. Oxford University Press, New York.

PETCHEY, O. \& GASTON, K.J., 2006.- Functional Diversity: Back to Basics and Looking Forward. Ecology Letters, 9: 741-758.

POT MUNICIPIO DE BUENAVENTURA., 2002.- Plan de ordenamiento territorial del municipio de Buenaventura. Alcaldía Municipal y cartografía. Ministerio del Medio Ambiente. Buenaventura, Colombia.

QUIMBAYO, M.A., 2006.- Caracterización de fauna silvestre Centro forestal tropical Bajo Calima (en) Los bosques secundarios del trópico húmedo colombiano: caso del Bajo Calima. Características y expectativas para el manejo sostenible y conservación de los ecosistemas boscosos del Bajo Calima, Valle del Cauca, Buenaventura, Colombia. Universidad del Tolima, Facultad de Ingeniería Forestal, Ibagué, Colombia.

RANGEL, J.O., 2004.- Amenazas a la biota y a los ecosistemas del Chocó biogeográfico: 881-1002 (en) Diversidad biótica IV. Instituto de Ciencias Naturales, Universidad Nacional de Colombia, Bogotá.

ROOSMALEN, M.G.M.V., 1985.- Fruits of the Guianan Flora. Institute of Systematic Botany, Utrecht University.

ROXBURGH, S., SHEA, K. \& WILSON, B., 2004.- The intermediate disturbance hypothesis: patch dynamics and mechanisms of species coexistence. Ecology, 85 (2): 359-371.

SALGADO, E., 2007.- Definición de Tipos Funcionales de Especies Arbóreas y Caracterización de su Respuesta a Diferentes Intensidades de Perturbación en un Bosque Muy Húmedo Tropical mesoamericano: Tesis de Maestría, Escuela de Posgrado, CATIE, Turrialba, Costa Rica.

THE PLANT LIST., 2012.- A Working List of all Plant Species. Disponible en: www.theplantlist.org

THOMPSON, I., 2011.- Biodiversidad, umbrales ecosistémicos, resiliencia y degradación forestal. Unasylva, 62 (2): 238.

THOMPSON, I., MACKEY, B., MCNULTY, S. \& MOSSELER, A., 2009.- Forest resilience, biodiversity, and climate change: a synthesis of the biodiversity/resilience/stability relationship in forest ecosystems. Secretaría del Convenio sobre la Diversidad Biológica, CBD Technical Series No. 43, Montreal, Canadá.

TILMAN, D., LEHMAN, C., 2002.- Biodiversity, composition, and ecosystem processes: theory and concepts: 365-370 (en) KINZIG, A.P., PACALA, W. \& TILMAN, D. (eds.) The functional consequences of biodiversity. Empirical progress and theoretical extensions. Princenton University Press, Princenton, New Yersey, EU.

VIOLLE, C., NAVAS, M., VILE, D., KAZAKOU, E., FORTUNEL, C., HUMMEL, I. \& GARNIER, E., 2007.- Let the concept of trait be functional. Oikos, 116: 882-892.

WALKER, B.H., 1992.- Biological diversity and ecological redundancy. Conservation Biology, 6: 18-23.

, 1995.- Conserving biological diversity through ecosystem resilience. Conserv. Biol., 9 (4): 747-752.

WALKER, B.H., KINZING, A. \& LANGRIDGE, J., 1999.- Plant attributes diversity, resilience, and ecosystem function: the nature and significance of dominant and minor species. Ecosystems, 2: 95-113.

WESTOBY, M. \& WRIGHT, J., 2006.- Land-plant ecology on the basis of functional traits. Trends in Ecology and Evolution, 21 (5): 261-268.

WRIGHT, S.J., 2003.- The myriad consequences of hunting for vertebrates and plants in tropical forests. Perspectives in Plant Ecology, Evolution and Systematics, 6 (1): 73-86. 
Apéndice 1. Composición florística de los bosques secundarios húmedos tropicales de Bajo Calima según THE PLANT LIST (2012).

\begin{tabular}{|c|c|c|c|c|}
\hline \multirow[t]{2}{*}{ Nombre vulgar } & \multirow[t]{2}{*}{ Familia } & \multirow[t]{2}{*}{ Nombre científico } & \multicolumn{2}{|c|}{ Sitio } \\
\hline & & & Conservado & Intervenido \\
\hline Peine mono & MALVACEAE & Apeiba glabra Aubl. & & $\mathrm{X}$ \\
\hline NR & PRIMULACEAE & Ardisia sp. & & $\mathrm{X}$ \\
\hline Costillo & APOCYNACEAE & $\begin{array}{l}\text { Aspidosperma desmanthum Benth. ex } \\
\text { Müll. Arg. }\end{array}$ & & $\mathrm{X}$ \\
\hline $\begin{array}{l}\text { Costillo } \\
\text { acanalado }\end{array}$ & APOCYNACEAE & Aspidosperma sp. & & $\mathrm{X}$ \\
\hline Jigua negro & LAURACEAE & Beilschmiedia sp.2 & & $\mathrm{X}$ \\
\hline Mare & MORACEAE & Brosimum rubencens Taub. & $\mathrm{X}$ & $\mathrm{X}$ \\
\hline Aceite María & CLUSIACEAE & Calophyllum brasiliense Cambess. & & $\mathrm{X}$ \\
\hline Tangare & MELIACEAE & Carapa guianensis Aubl. & $\mathrm{X}$ & $\mathrm{X}$ \\
\hline Canelo & LAURACEAE & Caryodaphnopsis sp. & & $\mathrm{X}$ \\
\hline Pacó & OCHNACEAE & Cespedesia macrophylla Seem. & & $\mathrm{X}$ \\
\hline Zanquiaraña & CLUSIACEAE & Chrysochlamys conferta Cuatrec. & & $\mathrm{X}$ \\
\hline Manglillo & CLUSIACEAE & $\begin{array}{l}\text { Tovomitopsis membranacea (Planch. \& } \\
\text { Triana) D'Arcy }\end{array}$ & $\mathrm{X}$ & \\
\hline Zanquiaraña & CLUSIACEAE & Chrysochlamys sp. & & $\mathrm{X}$ \\
\hline Caimito & SAPOTACEAE & Chrysophilum sp.2 & $\mathrm{X}$ & $\mathrm{X}$ \\
\hline Carbonero & CHRYSOBALANACEAE & Couepia sp.1 & $\mathrm{X}$ & \\
\hline Carbonero & CHRYSOBALANACEAE & Couepia sp.2 & $\mathrm{X}$ & \\
\hline Costillo negro & EBENACEAE & Diospyros sp. & & $\mathrm{X}$ \\
\hline Bagatá & PAPILIONACEAE & Dussia macrophyllata (Don.Sm) Harms & $\mathrm{X}$ & $\mathrm{X}$ \\
\hline Bagatacillo & PAPILIONACEAE & Dussia sp. & & $\mathrm{X}$ \\
\hline Caimito popa & SAPOTACEAE & Pradosia cuatrecasasii (Aubrév.) T.D. Penn. & $\mathrm{X}$ & $\mathrm{X}$ \\
\hline Caimito & SAPOTACEAE & Ecclinusa ramiflora Mart. & $\mathrm{X}$ & \\
\hline Ruda & ERYTHROXYLACEAE & ErytHroxylum sp. & $\mathrm{X}$ & \\
\hline Guasco & LECYTHIDACEAE & Eschweilera coriacea (DC.) S.A. Mori & $\mathrm{X}$ & \\
\hline Guasco peo & LECYTHIDACEAE & $\begin{array}{l}\text { Eschweilera integrifolia (Ruiz \& Pav. ex } \\
\text { Miers) R. Knuth }\end{array}$ & $\mathrm{X}$ & $\mathrm{X}$ \\
\hline Guasco blanco & LECYTHIDACEAE & Eschweilera panamensis Pittier & $\mathrm{X}$ & \\
\hline Guasco blanco & LECYTHIDACEAE & Eschweilera reversa Pittier & & $\mathrm{X}$ \\
\hline Rayado & EUPHORBIACEAE & Euphorbiaceae 1 & $\mathrm{X}$ & \\
\hline Mora amargo & RUBIACEAE & Faramea surrensis (Domn. Sm.) Domn. Sm. & & $\mathrm{X}$ \\
\hline Chaquiro & GOUPIACEAE & Goupia glabra Aubl. & & $\mathrm{X}$ \\
\hline $\begin{array}{l}\text { Cargadero } \\
\text { blanco }\end{array}$ & ANNONACEAE & Guatteria calimensis R.E. Fr. & & $\mathrm{X}$ \\
\hline Cargadero & ANNONACEAE & Guatteria cargadero Triana \& Planch. & $\mathrm{X}$ & $\mathrm{X}$ \\
\hline Castańo & MORACEAE & Helianthostylis sprucei Baill. & $\mathrm{X}$ & $\mathrm{X}$ \\
\hline Castańo & MORACEAE & $\begin{array}{l}\text { Helicostylis tovarensis (Klozsch y H. Karst.) } \\
\text { C.C. Berg }\end{array}$ & & $\mathrm{X}$ \\
\hline Guabo querre & CESALPINACEAE & Heterostemon sp. & $\mathrm{X}$ & \\
\hline Jigua & LAURACEAE & Indeterminado & & $\mathrm{X}$ \\
\hline NR & Indeterminado & Indeterminado 1 & $\mathrm{X}$ & \\
\hline NR & Indeterminado & Indeterminado 2 & $\mathrm{X}$ & \\
\hline Angarillo & Indeterminado & Indeterminado 3 & $\mathrm{X}$ & \\
\hline Guabo & MIMOSACEAE & Inga acreana Harms. & $\mathrm{X}$ & \\
\hline Guabo & MIMOSACEAE & Inga ingoides (Rich.) Willd. & $\mathrm{X}$ & \\
\hline Guabo & MIMOSACEAE & Inga sp.1 & $\mathrm{X}$ & $\mathrm{X}$ \\
\hline
\end{tabular}




\begin{tabular}{|c|c|c|c|c|}
\hline Jaboncillo & RUBIACEAE & Isertia pittieri (Standl.) Standl. & & $x$ \\
\hline Guasco nato & LECYTHIDACEAE & Lecythis minor Jacq. & $\mathrm{X}$ & \\
\hline $\begin{array}{l}\text { Carbonero } \\
\text { cáscara de huevo }\end{array}$ & CHRYSOBALANACEAE & Licania micrantha Miq. & $\mathrm{X}$ & $X$ \\
\hline Carbonero & CHRYSOBALANACEAE & Licania sp.1 & $\mathrm{X}$ & $x$ \\
\hline $\begin{array}{l}\text { Carbonero } \\
\text { sangrenito }\end{array}$ & CHRYSOBALANACEAE & Licania sp. 2 & $\mathrm{X}$ & \\
\hline Azulito & EUPHORBIACEAE & Mabea chocoensis Croizat. & $\mathrm{X}$ & $x$ \\
\hline Caimito silvador & SAPOTACEAE & Manilkara bidentata (A.DC.) A. Chev. & $\mathrm{X}$ & \\
\hline Casposo & MELASTOMATACEAE & Miconia ruficalyx Gleason. & $\mathrm{X}$ & \\
\hline Mora & MELASTOMATACEAE & Miconia sp.1 & & \\
\hline Mora & MELASTOMATACEAE & Miconia sp.3 & & $x$ \\
\hline Mora & MELASTOMATACEAE & Miconia sp.4 & $\mathrm{X}$ & \\
\hline Mora rojo & MELASTOMATACEAE & Miconia sp. 5 & $\mathrm{X}$ & \\
\hline Guayabillo & MYRTACEAE & Myrcia 1 & $\mathrm{X}$ & \\
\hline Jigua amarillo & LAURACEAE & Ocotea sp. & $\mathrm{X}$ & \\
\hline Palma mil pesos & ARACACEAE & Oenocarpus bataua Mart. & $\mathrm{X}$ & \\
\hline $\begin{array}{l}\text { Palma don } \\
\text { pedrito }\end{array}$ & ARACACEAE & Oenocarpus sp. & $\mathrm{X}$ & \\
\hline $\begin{array}{l}\text { Cuángare } \\
\text { chucha }\end{array}$ & MYRISTICACEAE & $\begin{array}{l}\text { Osteophloeum platyspermum (Spruce ex A. } \\
\text { DC.) Warb. }\end{array}$ & $\mathrm{X}$ & V \\
\hline $\begin{array}{l}\text { Cuángare } \\
\text { castaño }\end{array}$ & MYRISTICACEAE & Otoba sp.1 & & $X$ \\
\hline Cuángare indio & MYRISTICACEAE & Otoba sp.2 & $\mathrm{X}$ & $x$ \\
\hline Rayado & ANNONACEAE & Oxandra panamensis R.E. Fr & & \\
\hline Guabo vaina & MIMOSACEAE & Parkia velutina Benoist & $\mathrm{X}$ & \\
\hline Tostado & EUPHORBIACEAE & Pausandra sp. & & v \\
\hline Dormilón & MIMOSACEAE & Pentaclethra macroloba (Willd.) Kuntze. & $\mathrm{X}$ & \\
\hline Uvo & URTICACEAE & $\begin{array}{l}\text { Pourouma bicolor subsp. Chocoana (Standl.) } \\
\text { C.C. Berg y Heusden }\end{array}$ & $\mathrm{X}$ & \\
\hline Caimito & SAPOTACEAE & Pouteria buenaventurensis (Aubrév.) Pilz. & & $\gamma$ \\
\hline Caimito & SAPOTACEAE & Pouteria chocoensis (Aubrév.) T.D. Penn. & & \\
\hline Caimo guatín & SAPOTACEAE & Pouteria sp. 1 & & \\
\hline Caimito & SAPOTACEAE & Pouteria sp. 3 & & \\
\hline $\begin{array}{l}\text { Caimito } \\
\text { trapichero }\end{array}$ & SAPOTACEAE & Pouteria sp. 4 & $\mathrm{X}$ & \\
\hline $\begin{array}{l}\text { Caimito pros } \\
\text { pro }\end{array}$ & SAPOTACEAE & Pouteria sp. 5 & $\mathrm{X}$ & \\
\hline Anime blanco & BURSERACEAE & Protium panamense (Rose) I.M. Johnst. & $\mathrm{X}$ & \\
\hline Anime & BURSERACEAE & Protium ravenii D.M. Porter. & $\mathrm{X}$ & \\
\hline Cascajero & VOSCHYSIACEAE & Qualea lineata Stanfleu. & $\mathrm{X}$ & \\
\hline Madrońo & CLUSIACEAE & Garcinia madruno (Kunth) Hammel & & \\
\hline Juana se va & LINACEAE & Roucheria monsalveae A. H. Gentry. & $\mathrm{X}$ & \\
\hline Borojocillo & RUBIACEAE & Rubiaceae 1 & & \\
\hline Chanúl & HUMIRIACEAE & Sacoglottis ovicapa Cuatrec. & $\mathrm{X}$ & \\
\hline Garzo & SIMAROUBACEAE & Simarouba amara Aubl. & & \\
\hline Pacora & MALVACEAE & Sterculia speciosa K. Schum. & & \\
\hline Hueso & PAPILIONACEAE & Swartzia colombiana (R. S. Cowan) Torke. & $\mathrm{X}$ & \\
\hline Nn2 & PAPILIONACEAE & Swartzia sp.1 & & \\
\hline Machare & CLUSIACEAE & Symphonia globulifera L.F. & $\mathrm{X}$ & \\
\hline Manglillo & CLUSIACEAE & Tovomita sp. & $\mathrm{X}$ & \\
\hline Palo tunda & EUPHORBIACEAE & Tethrochidium sp. & & \\
\hline Bacao & MALVACEAE & Theobroma bicolor Humb. y Bonpl. & & \\
\hline
\end{tabular}




\begin{tabular}{|c|c|c|c|c|}
\hline $\begin{array}{l}\text { Cuángare punta } \\
\text { de lanza }\end{array}$ & MYRISTICACEAE & Virola Sebifera Aubl. & $\mathrm{X}$ & $\mathrm{X}$ \\
\hline Sangre gallina & HYPERICACEAE & $\begin{array}{l}\text { Vismia baccifera subsp. ferruginea (Kunth) } \\
\text { Ewan }\end{array}$ & & $\mathrm{X}$ \\
\hline Sorogá & VOSCHYSIACEAE & Vochysia ferruginea Mart. & $\mathrm{X}$ & $\mathrm{X}$ \\
\hline Pampanillo & VOSCHYSIACEAE & Vochysiacea 1 & $\mathrm{X}$ & $\mathrm{X}$ \\
\hline Palma amarga & ARACACEAE & Welfia regia $\mathrm{H}$. Wendl. & & $\mathrm{X}$ \\
\hline Palma memé & ARACACEAE & $\begin{array}{l}\text { Wettinia quinaria (O.F. Cook \& Doyle) } \\
\text { Burret. }\end{array}$ & & $\mathrm{X}$ \\
\hline Ruda & RUTACEAE & $\begin{array}{l}\text { Zanthoxylum brisasanum (Cuatrec.) P.G. } \\
\text { Waterman. }\end{array}$ & & $\mathrm{X}$ \\
\hline TOTAL & & 93 & 54 & 70 \\
\hline
\end{tabular}

